\title{
Séparer science et littérature
}

Les ambivalences du physiologiste Emil du Bois-Reymond (1818-1896)

\section{Françoise Willmann}

\section{CpenEdition}

Journals

Édition électronique

URL : http://journals.openedition.org/aes/955

DOI : $10.4000 /$ aes.955

ISSN : 2258-093X

Éditeur

Laboratoire LISAA

Référence électronique

Françoise Willmann, « Séparer science et littérature », Arts et Savoirs [En ligne], 7 | 2016, mis en ligne le 12 décembre 2016, consulté le 19 avril 2019. URL : http://journals.openedition.org/aes/955 ; DOI :

10.4000/aes.955

Ce document a été généré automatiquement le 19 avril 2019.

Centre de recherche LISAA (Littératures SAvoirs et Arts) 


\section{Séparer science et littérature}

Les ambivalences du physiologiste Emil du Bois-Reymond (1818-1896)

\section{Françoise Willmann}

Emil du Bois-Reymond fut l'une des personnalités scientifiques les plus en vue de son temps, l'un des protagonistes majeurs de ce qu'on a appelé l'École allemande de physiologie, réunissant à la suite de leur maître Johannes Müller (1801-1858), «le plus illustre physiologiste allemand de cette période $»^{1}$ outre E. du Bois-Reymond lui-même, Hermann Helmholtz (1821-1894), Carl Ludwig (1816-1895) et Ernst Brücke (1819-1892). Du Bois-Reymond passe communément pour être le fondateur de la physiologie scientifique. Il joua également un rôle de premier plan dans l'organisation institutionnelle de la science, notamment en tant que titulaire de la chaire de physiologie à Berlin à laquelle il accéda après le décès de Johannes Müller, en tant que recteur de l'université, une charge qu'il assuma à deux reprises, en tant que président de l'Académie royale des sciences de Berlin. Dans ces fonctions, il prononça nombre de conférences et discours dont beaucoup eurent un écho bien au-delà du cercle de ses confrères. Il marqua ainsi notablement les représentations collectives de la science au XIX ${ }^{\mathrm{e}}$ siècle.

2 Après avoir fréquenté le lycée français de Berlin, pourvu d'une solide culture classique, il s'engagea dans des études de mathématiques et de sciences, suivit également des cours de théologie et de philosophie, puis s'orienta vers la médecine en $1839^{2}$. Il soutint sa thèse en 1843 , mais sa vocation n'était pas la pratique de l'art médical. Dès 1841, il s'était lancé dans les travaux de physiologie qui allaient déterminer sa carrière, lorsque Johannes Müller lui proposa de reprendre et de poursuivre les recherches de l'Italien Matteucci (1811-1868) dont l'Essai sur les phénomènes électriques des «Animaux» venait de paraître à Paris en 1840.

Dans ses travaux, Du Bois-Reymond étudie les courants électriques dans les muscles et les nerfs. Pour lui, le vivant ne relève pas de forces spécifiques, contrairement à ce que soutiennent les vitalistes qu'il combat; il obéit à des lois qu'il importe de comprendre et de mettre en évidence grâce aux outils physiques et mathématiques. En 1875 et en 1877, Du Bois rassemble un certain nombre de ses articles en deux volumes intitulés Gesammelte Abhandlungen zur allgemeinen Muskel- und Nervenphysik. Mais ce sont les trois volumes des Untersuchungen über thierische Elektricität, parus respectivement en 1848, 1849 et 1884, qui 
retiendront tout particulièrement notre attention ici. La parution tardive du dernier volume, alors que la préface de mars 1848 annonçait déjà un plan détaillé que l'ensemble ne pourra respecter, indique combien Du Bois-Reymond tenait, malgré les difficultés rencontrées, à cette œuvre.

Les écrits de Du Bois-Reymond - discours, paratextes, écrits proprement scientifiques présentent des régimes d'écriture très différents, tantôt sobre, tantôt saturée d'images, voire de clichés, dont les constantes sont frappantes par-delà les décennies, révélatrices de la perception que les sciences de l'époque ont d'elles-mêmes, et étroitement liées à leur mode de fonctionnement, dont fait partie leur manière de s'écrire et de se communiquer. On lit souvent, sous la plume des commentateurs, que Du Bois-Reymond fut un défenseur combatif (streitbar) de sa discipline et de fait, sa verve polémique, soutenue par un usage pléthorique de métaphores, est un trait frappant de son écriture. Ces métaphores mobilisent d'une part l'image de la science qui doit se frayer son chemin, péniblement, dans la jungle déconcertante d'une nature encore impénétrable et accumule d'autre part les allusions multiples à la guerre de conquête dans laquelle sont engagés les savants. Cette dimension agonistique de la science chez Du Bois est explicite dans l'introduction aux Untersuchungen über thierische Electricität, mais imprègne également l'écriture du texte scientifique à proprement parler qui est à la fois historique, polémique, récit d'expériences et élaboration d'un savoir nouveau. Il mêle encore largement production scientifique et récit circonstancié de cette production, faisant apparaître le poids de la conflictualité dans la physiologie naissante, mais aussi la difficulté spécifique de Du Bois-Reymond à s'accommoder d'une écriture adéquate à son objectif, dépouillée d'affects et d'images et vouée à l'effacement du sujet. Cette alchimie particulière qui transforme du vivant, voire un affrontement avec le vivant, en une expression centrée sur l'énonciation de mesures, fonctions et lois, métamorphose son travail sur les grenouilles : le corps à corps avec les animaux s'estompe, disparaît même au cœur de la recherche, dans les récits des expériences et le développement des résultats. En revanche, la compétition avec les collègues, Matteucci en particulier, ressort tout particulièrement autour de deux préoccupations centrales: défendre la bonne manière de faire de la science et revendiquer la paternité de ses découvertes, face à un Matteucci dont Du Bois ne cesse de souligner les faiblesses et surtout la mauvaise foi. C'est ainsi que cette œuvre volumineuse reflète aussi bien la volonté et l'effort de scientificité que l'attachement de son auteur à quelque chose qui certes ne serait pas le roman de la science, mais qui en trahit tout de même la tentation, peut-être même le désir, hypothèse qui nous est suggérée par le discours de 1882, « Goethe und kein Ende » ${ }^{3}$, qui laisse transparaitre l'idée que se fait Du Bois-Reymond de la frontière entre science et littérature.

Lorsque Du Bois-Reymond introduit ses Untersuchungen über thierische Elektricität, il les situe dans le cadre de la compréhension du vivant. Il entend par ses travaux contribuer à infirmer la croyance en des «forces vitales" qui seraient propres aux phénomènes organiques. Du Bois récuse l'existence de telles forces dont il estime qu'elles relèvent de la métaphysique. Pour lui, organique et inorganique obéissent aux mêmes lois, lois que nous pouvons appréhender grâce à la physique et aux mathématiques. L'électricité est un de ces phénomènes qui ont donné lieu à de multiples interprétations irrationnelles et que la physique doit permettre de localiser et de clarifier. Parmi les travaux précurseurs dans ce domaine, Du Bois-Reymond reconnaît une dette particulière à l'égard d'Alexander von Humboldt (1769-1859) qui pratiqua d'innombrables expériences sur lui-même et sur une foule d'animaux, notamment sur des grenouilles. Du Bois, se servant d'instruments qu'il 
n'a cessé de repenser et de perfectionner ainsi que des outils de la physique, a travaillé durant près de quatre décennies à la frontière entre le vivant et le mort, ses expérimentations portant tantôt sur l'animal vivant, tantôt sur l'animal que l'on vient de tuer, tantôt sur l'animal dit «indemne » (unversehrt) tantôt sur l'animal «préparé » ( zugerichtet).

6 Si Du Bois-Reymond exprime à l'occasion des réticences à faire souffrir, ou du moins la volonté de limiter cette souffrance, pour peu que les précautions recommandées ne mettent pas en péril l'efficacité du dispositif expérimental, l'acte de tuer quant à lui ne semble problématique que du point de vue technique. En revanche, il s'appesantit sur les épreuves avant tout de deux types, mais non sans rapport entre elles, traversées par le scientifique: les difficultés procédant de la nouvelle manière d'écrire la science d'une part, les difficultés à se frayer la voie vers le savoir d'autre part.

\section{De la somme, œuvre d'un individu, à l'article, étape dans des échanges accélérés}

7 Le paratexte des Untersuchungen über thierische Elektricität, préface et postface, est imprégné du malaise qu'éprouve le physiologiste face à une évolution majeure dans les sciences modernes à laquelle, à le lire, il ne se soumet que partiellement et difficilement, à savoir ce qu'on pourrait appeler le passage d'une écriture individuelle à une écriture collective, le passage de la rédaction d'une œuvre à la publication d'articles, par essence provisoires et inachevés. En fait, la science change de genre littéraire et ce qu'on peut lire avec une netteté particulière chez Du Bois-Reymond, au milieu du XIX ${ }^{e}$ siècle et avec une charge émotionnelle intense, ce sont les enjeux de cette évolution et ses difficultés. Ces trois volumes sur l'électricité animale n'étant pas des textes de vulgarisation mais s'adressant aux physiologistes, ses collègues, Du Bois-Reymond évoque donc, tout en disant « je », des problèmes qu'il peut supposer partagés.

La préface au premier volume retrace en effet l'histoire de la recherche présentée au lecteur, mais aussi de sa publication; avec la postface au troisième volume, elle entérine la résignation à renoncer à la somme - Du Bois suggère que son ouvrage inabouti, comportant 1925 pages, en est une trace ultime, une survivance - au profit de l'article scientifique, appelé à être hégémonique. Les explications de Du Bois quant à l'évolution de son travail, ses choix de publication, ses insatisfactions et ses compromis, peuvent se lire comme un fragment d'autobiographie du scientifique et de la science.

Lorsque $\mathrm{Du}$ Bois s'engage dans la voie proposée par Johannes Müller, il lui faut tout d'abord mettre en place les conditions d'observation et d'expérimentation. Il se heurte à l'absence d'outils, voire de lieux adéquats, il faut trouver les grenouilles, les conserver dans de bonnes conditions l'hiver, etc. - bref, il lui faut une année, dit-il, avant qu'il n'obtienne les premiers résultats. Le travail systématique ne pourra commencer qu'en 1842, mais il lui faut s'interrompre dès l'automne 1842, pour une période indéterminée : il se résigne alors à une première publication dont il expose les insuffisances :

À cette époque, mes résultats, si je les avais exposés dans le détail pour qu'ils soient compréhensibles, n'entraient déjà plus dans les limites d'un article de revue. Quant à les rédiger pour en faire un écrit à part entière, je n'en trouvai pas le loisir; le matériau disponible n'était ni assez mûr, ni assez travaillé. L'issue que je trouvai fut de publier dans un premier temps les faits dont il m'importait de me réserver la 
priorité, et je le fis sous une forme brève, sans remarques introductives, et sans description des dispositifs et des manières de pratiquer les expériences. ${ }^{4}$

Le peu de succès de cette première publication est à mettre au compte avant tout, selon $\mathrm{Du}$ Bois, de son écriture, de sa présentation, de sa rhétorique. C'est moins sa recherche même qui est en cause, que l'écriture lacunaire marquée par la précipitation et le manque de maturité du travail, dans l'urgence d'une situation de précarité, pour des raisons moins scientifiques que relevant de sa place sur l'échiquier de la recherche : il fallait prendre des mesures pour s'assurer la primauté de ses premiers résultats.

11 De juin 1843 à mars 1848, Du Bois se remet à ses recherches et s'y voue entièrement, puis publie son premier volume en se justifiant de recourir à un mode de présentation devenu inhabituel :

Ce faisant, je m'écarte beaucoup, je ne le sais que trop, de la manière désormais commune de publier des recherches scientifiques. Je n'oublie à aucun moment combien cela eût été plus profitable, pour le public de mes lecteurs, pour la cause elle-même et donc aussi pour moi-même, si j'avais pu coucher mes résultats par écrit, au fur et à mesure de ma progression, dans des revues. $(1, \mathrm{VII})$

C'est sous forme d'aveu, voire de regret, que Du Bois note le caractère dépassé de sa démarche, tout en revendiquant le privilège d'une démarche individuelle, dans un contexte où il faudra pourtant se plier aux règles de ce qu'on n'appelait pas encore la communauté scientifique, et qui se présente de fait comme une constellation d'individus divers, aux positions diverses, amenés tantôt à collaborer, tantôt à se combattre. D'ores et déjà, la voie normale est, comme Du Bois le souligne, la publication d'articles. Cependant, au moment où des circonstances privées le forçaient à s'interrompre, ses travaux étaient déjà trop volumineux, mais pas suffisamment aboutis. L'interruption l'empêche de les mener à bien, mais lui donne aussi du temps pour méditer, et avancer. Et la reprise de ses travaux l'entraîne, dit-il, dans un labyrinthe qu'il décrit à grand renfort d'images; une citation assez longue mais très représentative, permettra également de se faire une idée de l'écriture de Du Bois :

Mais dès que je recommençai à interroger la nature, je me trouvai comme happé par un tourbillon ininterrompu d'énigmes toujours nouvelles, n'offrant nulle part de point fixe, et qui ne me permit de prendre plus ou moins pied que bien plus tard. L'enchevêtrement des réalités en ce domaine est étonnant. Les possibilités d'erreurs sont démultipliées à l'infini. Les interprétations qui s'offrent pour chaque expérience singulière sont innombrables. Parfois, une situation ayant été examinée et réexaminée, on pouvait avoir l'impression de s'acheminer vers quelque chose comme une fragile expérience cruciale. On la pratique; mais bien souvent, les résultats restent ambigus, en raison des difficultés insurmontables provenant de la nature des parties animales. Ou alors, elle indique clairement une direction précise, et oublieux d'anciennes embûches, je me laissais gagner par un sentiment de sécurité, et voilà qu'un nouvel essai me chassait de la position conquise qu'à l'instant encore je croyais avoir affermie. C'est ainsi que je titubai, des années durant, dans une douloureuse perplexité, jusqu'à ce qu'enfin certaines caractéristiques sur lesquelles je tombais de manière répétée, commencent à résister, cristallisant peu à peu et sous une forme de plus en plus certaine et compréhensible, le matériau dont je disposais par ailleurs.

Aussi, si j'avais voulu me plier à la manière dont on pratique désormais la publication, je me serais heurté à l'un des deux écueils suivants : j'aurais couru le risque de me contredire souvent et de parcourir publiquement cet enchaînement inévitable d'erreurs et de déceptions; ou alors j'aurais dû me limiter à juxtaposer en une suite sans vie des faits singuliers, des membres d'un tout encore éparpillés. (1, VIII) 
13 C'est ainsi que s'explique la décision de prendre le temps de la maturation avant de s'exprimer publiquement. Les incertitudes, les mystifications, les erreurs sont avancées pour justifier le fait d'avoir dérogé à la règle déjà commune, à savoir la publication régulière de résultats sous forme d'articles dans des revues. Les métaphores qui traversent le texte s'appesantissent sur les difficultés de l'entreprise : le scientifique veut convoquer la nature devant son tribunal ${ }^{5}$, mais il est pris dans un tourbillon qui menace de l'engloutir. Il vit des années de précarité, toujours près de se noyer dans l'océan des réalités à décrypter, menant une recherche angoissée trop souvent vaine, à l'affût de certitudes auxquelles se raccrocher et qui sans cesse se dérobent ${ }^{6}$.

On ne peut manquer de confronter à cette préface de 1848 le discours que prononcera son auteur en 1872 à Leipzig, devant la société savante, la Gesellschaft Deutscher Naturforscher und Ärzte qui fêtait cette année-là ses 45 ans d'existence. Ce discours fit grand bruit, bien au-delà du cercle de ses pairs, notamment par sa chute provocatrice, Ignorabimus ${ }^{7}$. Il suffira ici d'en rappeler le début :
Messieurs,
Tout comme un conquérant des temps anciens pouvait, en un jour de repos interrompant ses campagnes victorieuses, éprouver le besoin que soient constatées plus précisément les frontières de l'immensité des terres soumises à sa domination, afin d'exiger son tribut de tel peuple non encore soumis à l'impôt, ou de repérer un désert, véritable obstacle naturel aux troupes de ses cavaliers, pour mieux connaître les bornes véritables de son pouvoir, de même, ce ne sera pas une entreprise indigne de la conquérante de notre temps, la science de la nature, que de tenter de cerner clairement, en un jour comme aujourd'hui, jour de repos et de fête, les véritables limites de son incommensurable empire. ${ }^{8}$

Vingt-cinq ans plus tard, la métaphore militaire déjà présente en 1848 , traduisant alors le point de vue du vaincu (le scientifique se désolait de ne pas parvenir à maintenir la position qu'il avait cru conquise) sert ici de point de départ triomphaliste : l'orateur campe avec une remarquable assurance la position de supériorité du vainqueur. La suite $\mathrm{du}$ discours a suscité d'innombrables controverses, et récemment encore, Gabriel Finkelstein développait l'idée que $\mathrm{Du}$ Bois-Reymond était au fond, en dépit des apparences, un sceptique. Mais qu'il ait voulu marquer les limites de la science ou qu'il ait au contraire prétendu en affirmer le pouvoir, on sera frappé par la posture guerrière ellemême, la tension conflictuelle permanente dans laquelle évolue le scientifique. Son imaginaire est imprégné de métaphores militaires, parce que la science est pour lui un combat dont l'issue est encore et restera incertaine, une lutte qui le met aux prises avec la nature, ses objets, ses instruments, ses collègues.

En 1848 donc, Du Bois-Reymond s'affiche en position de faiblesse. Il tente de justifier son recours à une écriture obsolète : au premier chef, la nature ne se laisse pas faire et la lutte que l'on est obligé de mener contre elle est éminemment périlleuse et visiblement anxiogène ; le deuxième argument avancé montre le savant aux prises avec ses confrères et concurrents : si la première publication, aussi inaperçue et rudimentaire qu'elle ait pu être, a du moins été lue par quelqu'un, c'est par l'auteur critiqué, Matteucci, lequel, affirme Du Bois-Reymond, ne l'a peut-être pas vraiment compris, mais l'a pillé. C'est donc la nécessité de protéger ses propres résultats et expérimentations de la concurrence qui, cette fois-ci, l'a fait renoncer à publier. Ainsi le scientifique se voit-il pris entre diverses contraintes, diffuser la science auprès des collègues tout en se préservant, assumer le caractère fragmentaire des résultats, jouer le jeu des échanges nécessaires, bref: 
préserver à la fois les intérêts propres et ceux de la recherche, et assumer l'aridité, voire l'ascèse qu'exige un travail extrêmement circonscrit.

17 Là aussi, c'est-à-dire face à l'objet même de sa recherche, l'imaginaire militaire sature le texte. Il justifie une tactique qui implique des renoncements bien pensés dans l'objectif de conquêtes durables. Du Bois assume la concentration sur le travail de détail qui implique de négliger d'assurer ses arrières et conduit à prêter le flanc aux attaques inopinées. Cela signifie également renoncer à se lancer dans de grandes expéditions et ne pas céder à l'attrait des buts lointains qu'on devine plus séduisants. Il revendique au contraire une méthode vouée à la consolidation de la position acquise, assurant le terrain, afin de favoriser les conquêtes ultérieures.

même temps que le souci de la stratégie adéquate, s'expriment pourtant les incertitudes liées aux insuffisances et à la vulnérabilité du chercheur lui-même. Du BoisReymond parle du souci qui le tourmente, craint de n'être pas à la hauteur des résultats obtenus. La grande quantité d'expériences, de résultats, n'est plus maitrisable; il lui arrive de refaire des expériences qu'il a oubliées. La masse des faits grandit, la complexité de l'ensemble également, de sorte qu'un pis-aller, la publication d'articles inaboutis, se révèle désormais une nécessité intrinsèque de la recherche. Et c'est ainsi qu'il en vient à se résigner à prendre parti pour une évolution dont tout son travail illustre, notamment en ce qu'il y déroge, combien il est amputation, mais aussi nécessité pour avancer, dans une entreprise qui, plus que jamais, est et sera collective.

Parmi toutes les données nouvelles que comporte l'entreprise scientifique, l'exigence du renoncement à l'ambition de parvenir tout seul aux résultats escomptés n'est en effet pas la moindre. Du Bois-Reymond aborde à travers ce récit en forme d'auto-justification les multiples aspects de la recherche scientifique et des interactions au sein de la recherche. Le concurrent est aussi un collaborateur et il ne faut pas négliger le risque de perdre le contact avec les autres, de s'enfoncer dans un solipsisme de la compréhension des phénomènes, ou tout simplement de dépasser le moment où les résultats atteints peuvent se communiquer :

Plus on s'isole longtemps du commerce avec l'extérieur, plus on se plonge dans un cercle de faits et d'observations connexes que l'on a soi-même créés, et plus on risque de perdre le point d'appui à partir duquel on peut les communiquer à ceux qui se trouvent en dehors de ce cercle. On s'habitue sans s'en rendre compte à donner à telle expression tel sens qui paraîtra complètement étranger à ces derniers et qui brisera la chaîne de la compréhension. (1, XIV)

En plus du danger qu'il y a à élaborer des faits que l'on tarde à partager, Du Bois-Reymond explicite ici la part prise, dans la tentative de saisir la nature, du travail sur le langage : il faut des mots nouveaux, pour les instruments inédits, pour les réalités qu'on pense observer ; ceux-ci ne sauraient se forger dans la solitude. Il faut redouter qu'augmente, au fur et à mesure que les connaissances s'accroissent, la difficulté à les communiquer. Se couper des collègues est donc impossible. Il faut se plier à une accélération des échanges, et veiller à la constitution d'un langage commun.

S'ajoute à cela la complexité des rapports internationaux entre les savants. Du BoisReymond, polyglotte - il cite en latin, français, italien, anglais - prend d'emblée la défense de la science allemande, ce qui n'est certes pas étranger aux constellations politiques de l'époque (qui affleurent à l'occasion dans la correspondance), mais reflète davantage le contexte de la rivalité scientifique même: les scientifiques allemands se heurtent à la suprématie parisienne du premier tiers du siècle ${ }^{9}$, et les échanges personnels ont été 
difficiles pour Du Bois, en dépit du soutien de la figure médiatrice que fut Alexander von Humboldt ${ }^{10}$.

\section{Repousser les attaques}

$\mathrm{Au}$ fur et à mesure que Du Bois pénètre dans sa recherche même, l'écriture se fait de plus en plus dépouillée. Les envolées lyriques et même les métaphores se font plus rares. Le récit scientifique se caractérise par une ascèse sur le plan de la manipulation du langage qui frappe d'autant plus qu'il apparaît comme le noyau d'un ensemble constitué de nombreuses couches.

$\mathrm{Du}$ Bois-Reymond commence en effet par situer son objet de recherche historiquement, tout en se démarquant de ceux qui trouveront une telle démarche superflue. Il défend l'idée qu'il faut tout d'abord délimiter son champ, identifier les phénomènes pertinents, distinguer les approches, repérer les avancées. Dans un discours de 1872, il déplorera d'ailleurs la disparition progressive de cette prise de recul historique dans les pratiques d'écriture scientifique ${ }^{11}$. On verra dès lors s'esquisser une histoire de conflits, controverses, confusions, stratégies plus ou moins désintéressées, qui permettent avant tout de montrer combien, à ce stade, l'électricité animale est peu comprise et encore plus mal étudiée. Les grands moments de l'histoire de l'électricité s'incarnent dans de grands noms, mais pour autant, les progrès sont loin d'être linéaires. Du Bois-Reymond est donc amené à entrer dans un dialogue souvent conflictuel avec ses prédécesseurs et contemporains, dont il analyse dans le détail, et à grand renfort de citations, erreurs et mérites. Il veille certes à adopter un ton mesuré et à contrebalancer les critiques par la mention des apports de chacun, mais il lui arrive de s'impatienter : ainsi, le physiologiste Gabriel Gustav Valentin (1810-1883), auquel il reprochait dès la préface un maniement selon lui contreproductif de l'outil mathématique, fait-il l'objet d'une diatribe particulièrement virulente. Ce faisant, son objectif, dit-il, est de « démasquer les ravages » exercés par Valentin «de manière éhontée » à travers son œuvre, "afin de prévenir l'influence délétère qu'une telle violence faite à la science, derrière le bouclier trompeur de ce nom, ne saurait manquer d'avoir à plus ou moins longue échéance.» (129) Cependant, c'est toujours le besoin de déterminer frontières et limites, afin d'éviter de se perdre dans la jungle des phénomènes et des approches trop peu fondées qui le taraude et que traduit l'emploi récurrent de l'idée d'errer, dans le double sens de (se) tromper et de s'égarer, à travers tous les composés et formes de irren, Irrtum, verwirren, verwirrt, Verwirrung, entwirren : se tromper, erreur, embrouiller, troublé, confusion, démêler.

Les considérations proprement méthodologiques s'appuient d'abord sur un dispositif instrumental rigoureux dont la mise en place exige une vigilance théorique de chaque instant, un soin infini dans les manipulations, mais aussi une grande créativité technique ${ }^{12}$. On quitte alors le pathos et rares sont les passages qui sont encore saturés de métaphores comme les citations mentionnées plus haut. L'écriture paraît au contraire singulièrement dépouillée, et pourtant, aussi discrète qu'en soit l'expression, le sentiment d'être menacé de toute part par des "attaques» ressurgit. C'est ainsi qu'il écrit, en présentant la manière dont il va examiner les "parties animales" à l'aide de son multiplicateur pour voir si elles développent de l'électricité, et après avoir précisé que les difficultés rencontrées par la mise au point du dispositif instrumental ne sont rien, comparées à celles qui le guettent désormais : 
La nature de celles-ci [les difficultés rencontrées jusqu'ici] fait que l'observateur doit s'attendre à tout moment à des attaques renouvelées de la part de celles-ci [les difficultés à venir]; des attaques qui la plupart du temps sont d'autant plus difficiles à repousser ${ }^{13}$ que les causes dont elles procèdent restent pour la plupart inconnues. $(1,123)$

Bois-Reymond, pourtant peu avare, comme on le voit, de justifications, n'exprime de scrupule à travailler quotidiennement sur un monceau de cadavres. Certes, quelques phrases ou mots lui échappent parfois, ainsi une citation de Alexander von Humboldt dont il reprend une formulation compatissante pour ces malheureux batraciens ; il juge de mauvais goût une anecdote mettant en scène la dissection brutale d'une souris ; il évoque avec réprobation un collègue qui se rend coupable d'un «bain de sang » inutile. Mais ce sont les insuffisances techniques et théoriques de cette expérience critiquable qu'il développe ${ }^{15}$. Globalement, l'écriture, concentrée sur la minutie et le soin apporté aux procédures, produit une objectivation du matériau de l'expérimentation telle que disparaît jusqu'au côté sanguinolent de l'opération, absorbé dans la difficulté à maintenir la propreté du dispositif expérimental ${ }^{16}$.

\section{La recherche sur l'électricité animale, ou le roman de l'électrophysiologie}

es diverses manipulations, que ce soit sur des animaux morts ou vivants - on les assomme, empoisonne, écorche, dissèque de diverses manières, on coupe des morceaux de muscles, on isole des nerfs, sur des individus ou des piles, etc. - dirigent (et détournent) le regard du savant et du lecteur vers l'aiguille de l'instrument de mesure : reste-t-elle immobile, bouge-t-elle de telle ou telle manière, dans tel ou tel sens, 
régulièrement ou non, comment comprendre ses mouvements, etc.? Du Bois-Reymond apporte un soin tout particulier à la description de ses expérimentations, et se montre très attentif au moindre détail qui compromettrait leur reproductibilité. Enfin, l'énonciation des lois et la formulation de théories repoussent dans l'ombre le matériau dont on est parti. Mais elles sont des objectifs annoncés et sans cesse différés, car l'œuvre scientifique elle-même n'a pas vocation à s'achever. L'essentiel du texte est à la fois mise en scène de l'expérimentation et narration quasiment romanesque, au sens que Du Bois lui-même donne à ce genre. Sven Dierig, s'appuyant sur une vignette ornant la première édition des Untersuchungen, représentant Du Bois-Reymond lui-même en train d'officier dans son laboratoire, rappelle le souci esthétique du scientifique, lié à deux passions de ce dernier, le sport et la culture classique :

Avec cette double passion, Du Bois-Reymond allait au-delà de l'expérimentation comme simple pratique de vérification de la théorie et de l'hypothèse. L'expérimentation au laboratoire était art plastique, pratique épistémique et artistique ayant pour but la réussite esthétique autant que la fabrication du savoir physiologique. ${ }^{17}$

Ce geste esthétique est en effet perceptible dans l'ouvrage. En témoigne également le soin apporté aux planches, de la main de Du Bois lui-même et représentant les dispositifs expérimentaux. La réprobation occasionnelle à l'encontre des bains de sang inutiles est peut-être de même nature. Mais un autre rapprochement s'impose. Le 15 octobre 1882, Du Bois-Reymond, nouveau recteur de l'université de Berlin, prononçait un discours intitulé "Goethe und kein Ende", où il se penchait, après bien d'autres, sur la question de l'apport du savant Goethe à la science. Du Bois s'y montrait particulièrement sévère à l'égard de Goethe, regrettant, non sans provocation, que ses activités scientifiques lui aient fait perdre un temps précieux qu'il aurait mieux fait, selon lui, de consacrer à sa vocation de poète, plutôt que de se mêler de recherches qui auraient progressé aussi bien sans lui.

Partant d'une analyse du personnage de Faust, Du Bois déplore l'image dévalorisante qu'il donne de l'université et de l'homme de science. Il critique son idéalisation de l'action au détriment de la soif de connaissance. Il regrette les «inconséquences logiques » et les « monstruosités éthiques $»^{18} \mathrm{du}$ personnage, séducteur, criminel, et adoptant pour finir malgré toute sa culture et son savoir - les positions moyenâgeuses les plus régressives, fréquentant sorcières, diables, fantômes, qui sont autant de négations radicales de toute connaissance, voire de toute possibilité de connaître. Cette lecture du Faust I qui, sans être dépourvue de fondement, n'en est pas moins singulièrement réductrice, nous montre $\mathrm{Du}$ Bois-Reymond en train d'appliquer à l'œuvre littéraire les règles de la recherche scientifique, ses règles. Mesurant la pièce de Goethe à l'aune de la rigueur et de la cohérence rationnelles, il ignore la polysémie et le doute dont se nourrit la littérature.

Le véritable enjeu cependant de sa diatribe est de diagnostiquer la raison de l'incompétence profonde de Goethe en matière scientifique. Pour ce faire, il distingue deux types de description de la nature, la description mécanique et la description graphique (celle qu'on peut mettre au crédit du botaniste Goethe). Cette dernière, appliquée à quelque chose d'organique, la feuille d'un arbre par exemple, ignorera les forces qui lui donnent sa forme; en revanche, la description mécanique, d'un système tel un ménisque capillaire, cherchera à aller jusqu'aux causes et forces dont découlent formes et mouvements. Quel que soit le statut qu'on voudra donner à ces causes et forces, le fait d'y ramener un phénomène satisfait notre besoin de causalité et nous arrache au 
sentiment douloureux de confusion que nous éprouvons face à la complexité incomprise du réel, Du Bois utilisant ici les termes récurrents déjà rencontrés Verwirrung et verwirren. Il faut croire que c'est la nature de notre intellect qui nous pousse à cette opération consistant à décomposer les phénomènes, et c'est précisément, selon Du Bois-Reymond, le sens de cette " causalité mécanique » qui échappe à Goethe. Or, on peut distinguer dans la narration littéraire un versant en quelque sorte complémentaire de cette démarche analytique, un volet constructif dans la composition d'une intrigue romanesque. Et c'est là que la faiblesse diagnostiquée de Goethe se confirme, car s'il est hors de question de douter des qualités exceptionnelles du poète, voire du conteur, Du Bois s'autorise tout de même une nuance critique :

Aussi incomparable qu'il [Goethe] ait pu être en tant que conteur, on regrette qu'il n'ait pas eu le don, certes secondaire mais néanmoins estimable, de concevoir une intrigue qui a du sens, de la compliquer plus ou moins grâce à ses artifices, d'intensifier la confusion jusqu'à une apparente perplexité, pour la résoudre enfin, à son point culminant, de manière inattendue et plaisante. Ce manque aurait-il eu un rapport avec le fait que Goethe ne prenait plaisir, ni aux subtilités de l'analyse ni aux dispositifs expérimentaux complexes ? ${ }^{19}$

Cependant, le modèle certes «secondaire mais néanmoins estimable » où Goethe déçoit, n'aurait-il pas quelque parenté avec celui qui structure les trois volumes des Untersuchungen über thierische Elektrizität, rédigés, eux, par quelqu'un qui, visiblement, «prenait plaisir [...] aux subtilités de l'analyse» autant qu'aux «dispositifs expérimentaux complexes »?

Car les études d'électrophysiologie de Du Bois ne sont pas un manuel ${ }^{20}$. Elles se présentent, on l'a vu, sous la forme d'un récit très complexe dont le point de départ, une "définition conceptuelle ", consiste à baliser le vaste terrain qu'il s'agit de défricher. Ce faisant, l'auteur convoque les protagonistes anciens et récents de cette branche encore neuve de la physique organique, dont la plupart seront les personnages de sa recherche. Dans le premier volume, la partie historique tient une place particulièrement importante, le retour sur ses prédécesseurs occupant 150 pages, avant qu'il n'en vienne aux questions précises de méthode. Pour autant, les personnages évoqués ne tardent pas à revenir sur le devant de la scène, car si Du Bois se met en devoir d'exposer ses approches et ses instruments, cela le ramène presque immédiatement aux conceptions et pratiques des autres acteurs de son récit qui devient également une histoire des instruments, de leurs caractéristiques, de leurs usages, de leurs évolutions et améliorations, incarnée dans celle des savants qui s'en sont servis, de leurs avancées et de leurs erreurs, de leurs certitudes et de leurs tâtonnements. Et il en ira de même dans les phases suivantes, manipulation des animaux, expérimentation elle-même, études à proprement parler du courant musculaire et nerveux. Ainsi, au fil des pages, le récit de ces études d'électrophysiologie s'avère-t-il une véritable mise en scène de leurs méandres, le scientifique conteur recourant plus d'une fois au suspense, différant la solution d'un problème soulevé tantôt par un manque de connaissances, tantôt par des erreurs, les siennes ou celles de ses concurrents.

$\mathrm{Au}$ fond, il s'agit pour Du Bois de faire revivre à son lecteur les tourments de la confusion et des errances contre lesquels il n'a cessé de lutter, pour le mener progressivement vers les sommets de la connaissance scientifique. Mais ce n'est pas lui qui conçoit, complique et résout, et la progression n'atteindra pas son point culminant. L'œuvre reste un «monstrum per defectum» $(3,501)$. Et parmi les protagonistes de son récit, c'est le prédécesseur et concurrent Matteucci qui tient une place essentielle. Du Bois ne cesse de 
se confronter à lui, lui reprochant essentiellement absence de rigueur et malhonnêteté, dans tous les domaines :

Partout la même déloyauté, à l'égard de la nature, de lui-même, et des autres chercheurs ; la même légèreté dans les affirmations et les hypothèses avancées, qui semblent davantage destinées à attirer l'attention qu'à épouser les faits; la même désinvolture dans les conclusions qui l'empêche de pousser plus loin les avantages dont le hasard l'a plus d'une fois comblé au cours de ses longues recherches menées avec une remarquable ardeur, enfin, une négligence au niveau de la forme que l'on ne peut excuser par l'utilisation d'une langue qui lui était étrangère, puisqu'elle s'exprime davantage dans une mauvaise organisation de sa matière et dans l'imprécision ambiguë de l'expression [...]. $(1,113)$

On voit que ce qui indispose profondément Du Bois-Reymond est ce dont nous l'avons vu souffrir tout au long de son travail, la confusion, à laquelle il faut opposer son effort long et patient de rigueur. Et c'est en filigrane - l'accusation de malhonnêteté en moins tout de même - ce qui provoque son irritation contre le poète Goethe qui tenait à être savant également. Reconnaître que ce dernier peut se prévaloir d'intuitions ou d'aperçus féconds ne lui donne pas le droit de prétendre au titre de scientifique, ou du moins ne permet à personne de le lui accorder. Ainsi, ceux qui en font un précurseur de Darwin ignorent-ils que ce n'est pas l'idée qui fait le progrès scientifique, mais le travail long, aride, minutieux, voire ingrat, comme en témoignent « les spécialistes qui, au prix d'un travail acharné, se fraient un chemin à travers l'enchevêtrement (Gewirr) des détails »"12.

Les Untersuchungen über thierische Elektrizität sont le récit d'un tel travail acharné, qui se rapproche du manuel; il renseigne sur l'évolution d'une discipline, des difficultés spécifiques de son émergence et de son avancement. Au-delà de cette dimension quasiment technique, apparaît également une réflexion plus large sur le fonctionnement de la science, notamment, en creux, le souci d'une éthique de la recherche. Mais Du Bois prend garde aussi - encore pourrait-on dire - à la dimension rhétorique de sa présentation. Un narrateur raconte une histoire, l'histoire scrupuleusement construite d'une recherche qui est aussi celle de différents acteurs, dont les interactions mobilisent également des émotions, certaines très privées, admiration, jalousie, indignation, d'autres plus universelles, déceptions, espoirs. Parmi ces émotions, où frappe la prééminence des tensions douloureuses (la carrière de Du Bois-Reymond en revanche ne se résume pas aux conflits, loin de là̀22), on voit la récurrence de l'idée que la science exige des sacrifices permanents. Le jugement sur Goethe trouve son expression peut-être la plus intéressante dans les phrases suivantes :

Du darwinisme qui, par la génération spontanée confine à la théorie de Kant et Laplace, de la naissance de l'homme du chaos par le jeu des atomes déterminé mathématiquement d'éternité en éternité, de la fin du monde par la glaciation - de toutes ces visions que notre génération regarde en face avec insensibilité, de même qu'elle s'habitue aux frayeurs du train - de tout cela, Goethe se serait détourné avec horreur. ${ }^{23}$

La science est conquête, mais tout autant renoncement, et Du Bois le formule ici avec une rare brutalité. Lui-même est prêt, visiblement, à accepter la réduction à l'atome, la fin de l'univers, l'abandon de toute consolation métaphysique, et en tire un orgueil perceptible entre les lignes (et n'interroge pas cette prise de pouvoir sur l'humanité, pas davantage que celle sur les animaux dont il se sert). En revanche - et l'on peut supposer que c'est là la clé de la publication d'un ouvrage qualifié par lui-même d'anachronique, autant que de son animosité à l'égard de Goethe - il n'est pas encore prêt à l'effacement du sujet qu'implique le travail scientifique. Aussi, tout en défendant une science expérimentale 
rigoureuse et mathématisée, il reste attaché à une écriture en décalage avec cet objectif et ses pratiques. Dans un texte scientifique, l'homme de science disparaît. L'imaginaire qui traverse les écrits d'Emil du Bois-Reymond d'une part (conflictualité, mise en scène des obstacles, angoisse de l'errance et des erreurs), les choix d'écriture d'autre part (dimension historique, présentation des expérimentations, dialogue/controverse avec ses concurrents) sont déterminés en dernier ressort par un réflexe de défense face à cet oubli programmé.

\section{NOTES}

1. Mirko D. Grmek (ed.), Histoire de la pensée médicale en Occident, vol. 3, Du romantisme à la science moderne, Paris, Seuil, 1999, p. 23. Voir aussi Gunter Mann (ed.), Naturwissen und Erkenntnis im 19. Jahrhundert : Emil du Bois-Reymond, Hildesheim, Gerstenberg Verlag, 1981.

2. La famille paternelle, originaire du canton de Neuchâtel, qui, jusqu'en 1848, était rattaché à la Prusse, était tournée vers la culture française. Son père, Felix-Henri du Bois-Reymond, fut Conseiller secret au ministère des affaires étrangères à Berlin ; sa mère, fille d'un pasteur de la communauté française de Berlin, est une petite-fille du célèbre graveur Daniel Chodowiecki.

3. Emil du Bois-Reymond, Goethe und kein Ende, Berlin, Akademie der Wissenschaften, 1882. Le titre fait allusion à un texte de Goethe en l'honneur de Shakespeare, mais prend une coloration négative, et pourrait se traduire par « Pour en finir avec Goethe ».

4. Emil du Bois-Reymond, Untersuchungen über thierische Elektricität, («Études sur l'électricité animale ») Berlin, Verlag von G. Reimer, 1848, volume 1, p. VI. Les indications de page dans le texte renvoient à cet ouvrage en trois volumes. Dans tous les cas, c'est moi qui traduis (FW).

5. On entend la référence au célèbre passage de la deuxième préface à la Critique de la Raison Pure, où Kant affirme: «Il faut donc que la raison se présente à la nature [...] comme un juge en fonctions qui force les témoins à répondre aux questions qu'il leur pose. ", Immanuel Kant, Critique de la Raison Pure, traduction de A. Tremesaygues et B. Pacaud, Paris, PUF, p. 17.

6. Voir, à propos de la métaphore sous-jacente du naufrage, Hans Blumenberg, Naufrage avec spectateur: paradigme d'une métaphore de l'existence, traduction de Laurent Cassagnau, Paris, l'Arche, 1994.

7. Voir Kurt Bayertz, Myriam Gerhard, Walter Jaeschke (ed.) Weltanschauung, Philosophie und Naturwissenschaft im 19. Jahrhundert, Band 3 : Der Ignorabimus-Streit, Hamburg, Felix Meiner 2007 ; K. Bayertz, M. Gerhard, W. Jaeschke (ed.), Der Ignorabimus-Streit, Hamburg, Felix Meiner, 2012.

8. K. Bayertz, M. Gerhard, W. Jaeschke (ed.), Der Ignorabimus-Streit, Hamburg, Felix Meiner, 2012, p. 1.

9. Cf. l'étude classique de Joseph Ben-David, The scientist's role in society, a comparative study, New Jersey, Prentice-Hall, 1971.

10. Cf. Gabriel Finkelstein, «M. du Bois-Reymond Goes to Paris », British Journal for the History of Science 36, $\mathrm{n}^{\circ}$ 3, septembre 2003, p. 261-300. Cf. aussi la lettre de Hermann Helmholtz du 17 septembre 1850, qui donne le ton : «J'ai reçu le rapport de l'Académie [des sciences de Paris] sur tes expériences. On ne peut pas le dire autrement : il est rédigé avec un maximum de mauvaise volonté. " Suit une analyse qui aboutit à une mise en cause virulente de la compétence même des physiologistes français. Christa Kirsten (ed), Dokumente einer Freundschaft, Briefwechsel zwischen Hermann von Helmholtz und Emil du Bois-Reymond 1846-1894, Berlin, Akademie-Verlag, 1986, p. 105. 
11. "Über die Geschichte der Wissenschaft », 1872, in Siegfried Wollgast (ed.), Emil du BoisReymond, Vorträge über Philosophie und Gesellschaft, Hamburg, Felix Meiner, 1974.

12. Cf. Hans-Jörg Rheinberger, Michael Hagner (ed.), Die Experimentalisierung des Lebens, Experimentalsysteme in den biologischen Wissenschaften 1850/1950, Berlin, Akademie Verlag, 1993.

13. Il faut souligner la violence du terme employé par Du Bois : zurückschlagen.

14. Du Bois-Reymond emploie ici un terme récurrent dans le récit de la science de l'époque : Mühe.

15. Emil du Bois-Reymond, op. cit. (note 4) Volume 1, p. 233.

16. Cf. Françoise Willmann, «Le supplice des grenouilles, point aveugle du progrès scientifique. Les travaux d'Emil du Bois-Reymond. », in Aurélie Choné, Catherine Repussard (ed.) Recherches Germaniques, hors-série $n^{\circ} 10$, Des animaux et des hommes, Von Tieren und Menschen, Strasbourg, Presses universitaires de Strasbourg, 2015, p. 37-54.

17. Sven Dierig, Wissenschaft in der Maschinenstadt. Emil du Bois-Reymond und sein Laboratorium in Berlin, Göttingen, Wallstein, 2006, p. 132.

18. Emil du Bois-Reymond, op. cit. (note 3), p. 12.

19. Ibid., p. 21.

20. Lorsque Carl Ludwig s'engagera dans la rédaction de son manuel (Handbuch der Physiologie des Menschen, paru en deux tomes en 1852 et 1856), Du Bois-Reymond l'encouragera à ce travail dont il souligne la nécessité et en même temps le renoncement qu'il implique, exigeant de son auteur qu'il fasse passer sa propre recherche au second plan.

21. Emil du Bois-Reymond, op. cit. (note 4), p. 25.

22. L'importance des amitiés scientifiques dans sa carrière est un fait connu. En 1849, il devint président d'une société de physique (Physikalische Gesellschaft) fondée en 1845, dont le noyau était constitué de 5 personnes qui se réunissaient tous les quinze jours, à l'initiative de Du Bois, depuis 1841. Voir l'introduction à la correspondance entre Du Bois-Reymond et Humboldt: Ingo Schwarz, Klaus Wenig (ed.), Briefwechsel zwischen Alexander von Humboldt und Emil du Bois-Reymond, Berlin, Akademie Verlag, 1997.

23. Emil du Bois-Reymond, op. cit. (note 3), p. 25.

\section{RÉSUMÉS}

Emil du Bois-Reymond (1818-1894) a publié une part importante de ses travaux sur l'électricité animale en trois volumes intitulés Untersuchungen über thierische Elektricität, $(1848,1849,1884)$. Malgré ses quelque mille pages, l'ouvrage est inachevé et cela pour des raisons multiples relevant de l'évolution des modalités de production, de publication et d'écriture des sciences, une évolution à laquelle Du Bois-Reymond prend une part active tout en ne cessant de revenir sur la souffrance qu'elle implique. Son obstination à faire paraitre un ouvrage dont il affirme lui-même le caractère anachronique trahit la difficulté à se résigner à l'effacement du sujet qu'exige le travail scientifique, que l'on retrouve au cœur de son célèbre discours de 1882 sur Goethe, poète et savant, explorant les frontières entre science et littérature.

In Untersuchungen über thierische Elektricität $(1848,1849,1884)$, the physiologist Emil du Bois Reymond (1818-1894) attempted to bring together all his work on the electrical nature of nerve signals. The third volume, which was published in 1884 , remained unfinished because of pressure 
to publish articles rather than a cumbersome narrative. In commenting the nature of modern scientific work and writing, Du Bois-Reymond insisted on the difficulties and suffering brought about by the scientific work itself as well as by the writing up of the procedures and results. As one can see in his talk on Goethe (1882), whom he reproached for having wasted too much of his time trying to be a scientist, du Bois Reymond's hostility against the poet reveals his own reluctance to disguise the subject in accordance with the formal demands of a scientific narrative.

INDEX

Mots-clés : discours de savants, écriture de la science, erreurs et errances, frontières entre science et littérature

\section{AUTEUR}

\section{FRANÇOISE WILLMANN}

Université de Lorraine, CEGIL EA 3944/LHSP-Archives Poincaré UMR 7117 